\title{
Health Insurance Portability and Accountability Act
}

National Cancer Institute

\section{Source}

National Cancer Institute. Health Insurance Portability and Accountability Act. NCI

Thesaurus. Code C62504.

United States legislation enacted in 1996 that aims to provide health data privacy and security. 